\title{
DIVERSITY AND POPULATION STRUCTURE OF MACROINVERTEBRATES IN THE GLACIER FED STREAM BALKHILA AT TILFARA FROM GARHWAL HIMALAYA
}

\author{
Mohd Tariq*, Chandra Bhanu Kotnala and Anoop Kumar Dobriyal \\ Department of Zoology, HNB Garhwal University (A Central University), BGR Campus Pauri Garhwal- \\ 246001, Uttarakhand, India
}

*Corresponding Author Email id: mtariq.t94@gmail.com

Received: 28.09.2020; Revised: 19.11.2020; Accepted: 23.11.2020

(C)Society for Himalayan Action Research and Development

\begin{abstract}
This paper deals with the study of macrozoobenthic community in the glacier fed stream Balkhila which is an important tributary of Alaknanda River. In this study it was found that the maximum macrozoobenthos were recorded in the month of February $\left(387.5 \pm 106.5\right.$ units $\left./ \mathrm{m}^{2}\right)$ followed by January $\left(382.2 \pm 144.7\right.$ units $\left./ \mathrm{m}^{2}\right)$ whereas, minimum in July $\left(21.6 \pm 15.3\right.$ units $\left./ \mathrm{m}^{2}\right)$ and August $\left(21.6 \pm 0.0\right.$ units $\left./ \mathrm{m}^{2}\right)$. The stream is represented by 15 genera belonging to 6 orders viz. Ephemeroptera (Baetis, Cinygmula, Caenis, and Heptagenia), Trichoptera (Glossosoma, Rhyachophila, Agapetus, Hydropsyche, Chimarra and Stenopsyche), Diptera (Antocha and Chironomus), Coleoptera (Psephenus), Odonata (Euphaea) and Plecoptera (Perla). The regression analysis and the correlation coefficient were used to show the relationship between macrozoobenthos and various physico-chemical parameters. The Shannon-Wiener diversity index was calculated to study the benthic diversity of the stream. In order to depict the homogeneity among different benthic orders, the multivariate cluster analysis was used. The Canonical correspondence analysis (CCA) was used to investigate the seasonal effect of detrimental ecological factors on benthic population.
\end{abstract}

Key words: Balkhila stream, Macrozoobenthos, Diversity, CCA

\section{Introduction}

Benthic macroinvertebrates are mostly the nymphs and the larvae of insects in an aquatic ecosystem which inhabits stream bottom. They act as a natural source of food for fishes along with periphyton. Benthic macroinvertebrates are the common and most popular group of freshwater organisms which are used in assessing the water quality. They are very useful in biomonitoring although a practice for some well-balanced monitoring programs for their quantitative sampling and community analysis is required (Rosenberg and Resh, 1993). The functioning of river ecosystem is immensely contributed by benthic macroinvertebrates community. The nature of disturbance in the system can be assessed by their diversity and composition at any particular time (Stanford and Ward, 1983). The various physico-chemical parameters affect the community pattern and distribution of benthic macroinvertebrates. The altered water condition and thermal regime can have serious effect on cold stenothermic species as their growth, reproduction, metabolism, distribution and emergence is determined by temperature (Milner et. al., 2001).

Glacier fed streams are probably the harshest environments and therefore they usually show a characteristic spatial pattern in longitudinal distribution of diversity and assamblages of species downstream and away from the influence 
of the glacier (Saether, 1968; Steffan, 1971). Even though glacier fed streams are considered harsh environments and constrained habitats in nature with low spatial heterogeneity (IIg and Castella, 2006), there is considerable variations in assemblage composition and longitudinal diversity patterns among streams (Brittain et. al., 2001; Milner et el., 2001). The relationship among assemblages, longitudinal patterns and environment biota are all expected to be largely similar in closely situated streams. We assume that the stream macroinvertebrates within catchments are not strongly dispersal limited (Thompson and Townsend, 2006; Heino and Mykra, 2008).

In Uttarakhand, studies on benthic macroinvertebrates have been carried out by several researchers. Bahuguna et. al., (2004) studied the variations in the density composition and diversity of the benthic macroinvertebrates of the Ganga river in the up and downstream sections of the impounded zones. They found that the regulated stretch harbor only Ephemeroptera, Diptera, Trichoptera and Mollusca whereas, Plecopterans were absent while Coleopterans were very rare in the regulated stretch. Dobriyal et.al. (2009) has worked on the interactions of substratum heterogeneity with macrozoobenthos in the Eastern Nayar river. Katoch et.al. (2015) described population structure and diversity analysis of benthic Ephemeropterans in Western Nayar river. Balodi and Koshal (2015) studied the macrozoobenthos of Khoh stream and Koshal et al. (2017) analyse the communities structure of Macroinvertebrate in Rawasan Stream. whereas Kumar and Nautiyal (2019) studied the community feature of benthic macroinvertebrates of Bhagirathi river at three stations and suggested that urgent measures are required to improve the ecological health of the river. Bahuguna et.al. (2019) have reported the density and diversity of aquatic mites from spring fed tributaries of river Alaknanda.

The present study was designed to investigate the macrozoobenthos diversity and their relationship with various abiotic factors of the river Balkhila at Tilfara during November, 2019 to October, 2020 .

\section{Materials and Methods}

Study area: The Balkhila river is glacier fed and is located at higher altitude. The present study was carried out at Tilfara within a latitiude of $30^{\circ} 23^{\prime}$ $17^{\prime \prime} \mathrm{N}$ and longitude $79^{\circ} 19^{\prime} 15^{\prime \prime} \mathrm{E}$ and altitude 932 masl. The Tilfara spot is covered by some mixed vegetation (Fig. 1).

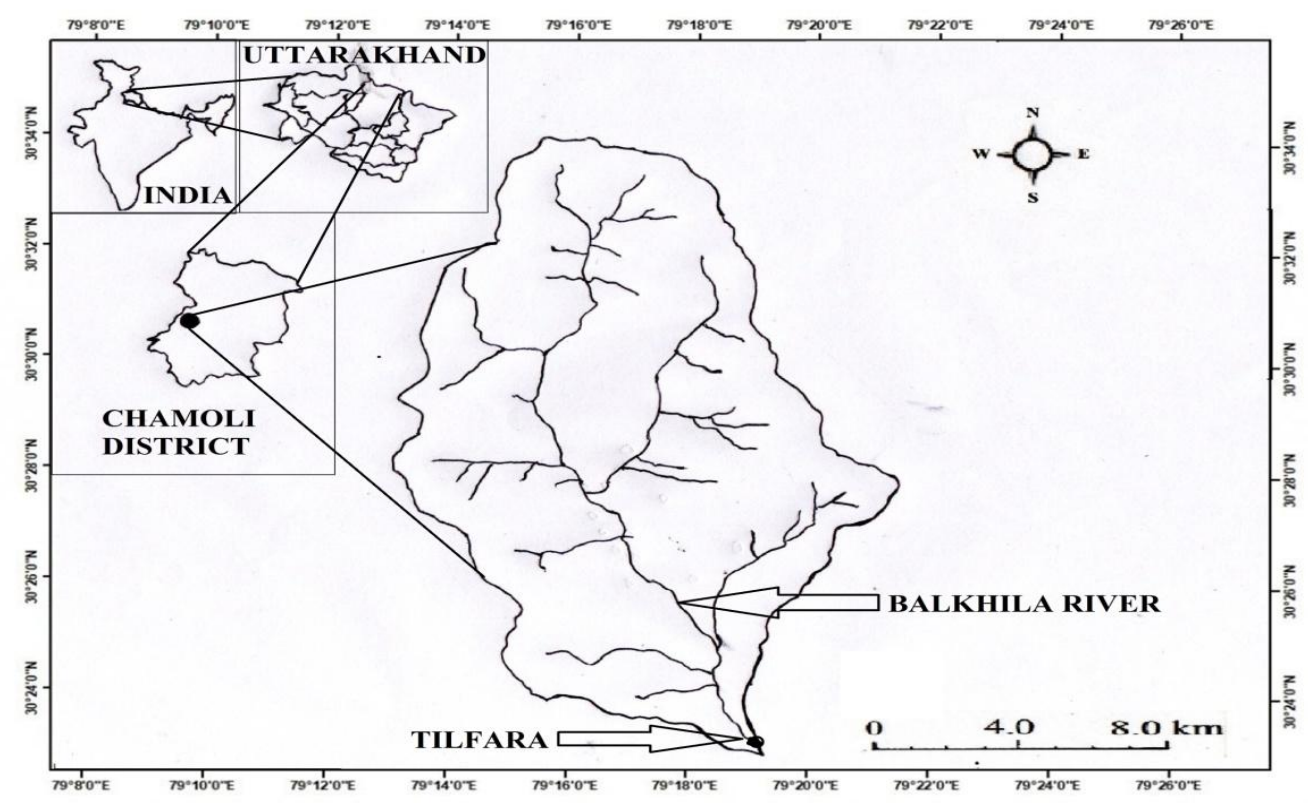

Figure 1: Location of sampling site of the stream Balkhila. 


\section{Physico-chemical Parameters analysis}

The physico-chemical parameters were analysed as per standard methods (Welch, 1948 and APHA, 2012). Surface water temperature was analyzed with the help of centigrade thermometer. The velocity of water current was estimated by float method. $\mathrm{pH}$ was recorded by portable electronic $\mathrm{pH}$ meter. Dissolved oxygen was analyzed by Winkler's method. Total hardness and total alkalinity was recorded by Welch method. The turbidity was analysed with the help of digital Nephelometer.

\section{Sampling of macrozoobenthos}

The macrozoobenthos were collected from $1 \mathrm{ft}^{2}$ by using serber sampler and picking with help of brush and forceps. The values were then converted into units. $\mathrm{m}^{2}$. The collected macrozoobenthos were preserved in $4 \%$ formalin and then carried to the laboratory. The identification was made with help of various keys and monograph (Edmondson, 1992; Hynes, 1970; Jessup et. al.,2002).

\section{Statistical Analysis}

The data was analysed by using various statistical tools. Regression analysis and correlation coefficient was used to present the relationship between physico-chemical parameters and total macrozoobenthos. The macrozoobenthos diversity was estimated by Shannon-Wiener diversity index $\left(\mathrm{H}^{\prime}=-\right.$ - pi.lnpi). The multivariate cluster analysis was used to show the hierarchical grouping among different orders of macozoobenthos. The canonical correspondence analysis (CCA) was used to show the seasonal effect of detrimental ecological factors on different macrozoobenthic orders. All the statistical analysis was made by using Microsoft excel and Past 3.0.

\section{Results and discussion}

Monthly variations in the physico-chemical parameters of the stream Balkhila at Tilfara during the year 2018-19 and 2019-20 is presented in the Table 1.

Table 1: Monthly variations in physico-chemical parameters of the stream Balkhila at (Tilfara) during the year 2018-19 and 2019-20.

\begin{tabular}{|c|c|c|c|c|c|c|c|}
\hline MONTHS & WT $\left({ }^{0} \mathrm{C}\right)$ & CV (m.sec ${ }^{-1)}$ & pH & DO (mg.l $\left.\left.\right|^{-1}\right)$ & TA (mg. $\left.\mathbf{l}^{-1}\right)$ & TH (mg.l-1) & T (NTU) \\
\hline NOV. & $\begin{array}{l}13.3^{\mathrm{a}} / 12.9^{\mathrm{b}} \\
(13.1 \pm 0.3)\end{array}$ & $\begin{array}{c}0.83^{\mathrm{a}} / 0.84^{\mathrm{b}} \\
(0.84 \pm 0.01)\end{array}$ & $\begin{array}{c}7.9^{\mathrm{a}} / 8.0^{\mathrm{b}} \\
(8.0 \pm 0.1)\end{array}$ & $\begin{array}{l}13.1^{\mathrm{a}} / 12.5^{\mathrm{b}} \\
(12.8 \pm 0.4)\end{array}$ & $\begin{array}{c}44.0^{\mathrm{a}} / 46.8^{\mathrm{b}} \\
(45.4 \pm 2.0)\end{array}$ & $\begin{array}{l}70.0^{\mathrm{a}} / 72.0^{\mathrm{b}} \\
(71.0 \pm 1.4)\end{array}$ & $\begin{array}{l}26.0^{\mathrm{a}} / 25.0^{\mathrm{b}} \\
(25.5 \pm 0.7)\end{array}$ \\
\hline DEC. & $\begin{array}{r}12.3^{\mathrm{a}} / 11.3^{\mathrm{b}} \\
(11.8 \pm 0.7)\end{array}$ & $\begin{array}{l}0.72^{\mathrm{a}} / 0.63^{\mathrm{b}} \\
(0.68 \pm 0.06)\end{array}$ & $\begin{array}{l}8.1^{\mathrm{a}} / 8.3^{\mathrm{b}} \\
(8.2 \pm 0.1)\end{array}$ & $\begin{array}{c}12.9^{\mathrm{a}} / 12.7^{\mathrm{b}} \\
(12.8 \pm 0.1)\end{array}$ & $\begin{array}{r}50.0^{\mathrm{a}} / 52.4^{\mathrm{b}} \\
(51.2 \pm 1.7)\end{array}$ & $\begin{array}{r}74.0^{\mathrm{a}} / 78.0^{\mathrm{b}} \\
(76.0 \pm 2.8)\end{array}$ & $\begin{array}{l}6.0^{\mathrm{a}} / 8.0^{\mathrm{b}} \\
(7.0 \pm 1.4)\end{array}$ \\
\hline JAN. & $\begin{array}{r}11.1^{\mathrm{a}} / 10.4^{\mathrm{b}} \\
(10.8 \pm 0.5)\end{array}$ & $\begin{array}{l}0.63^{\mathrm{a}} / 0.55^{\mathrm{b}} \\
(0.59 \pm 0.06)\end{array}$ & $\begin{array}{l}8.4^{\mathrm{a}} / 8.2^{\mathrm{b}} \\
(8.3 \pm 0.1)\end{array}$ & $\begin{array}{r}13.3^{\mathrm{a}} / 13.0^{\mathrm{b}} \\
(13.2 \pm 0.2)\end{array}$ & $\begin{array}{r}62.2^{\mathrm{a}} / 64.8^{\mathrm{b}} \\
(63.5 \pm 1.8)\end{array}$ & $\begin{array}{r}74.0^{\mathrm{a}} / 76.0^{\mathrm{b}} \\
(75.0 \pm 1.4)\end{array}$ & $\begin{array}{l}6.0^{\mathrm{a}} / 7.0^{\mathrm{b}} \\
(6.5 \pm 0.7)\end{array}$ \\
\hline FEB. & $\begin{array}{r}14.1^{\mathrm{a}} / 13.4^{\mathrm{b}} \\
(13.8 \pm 0.5)\end{array}$ & $\begin{array}{l}0.63^{\mathrm{a}} / 0.57^{\mathrm{b}} \\
(0.60 \pm 0.04)\end{array}$ & $\begin{array}{l}8.0^{\mathrm{a}} / 8.3^{\mathrm{b}} \\
(8.2 \pm 0.2)\end{array}$ & $\begin{array}{r}11.3^{\mathrm{a}} / 10.8^{\mathrm{b}} \\
(11.1 \pm 0.4)\end{array}$ & $\begin{array}{r}50.2^{\mathrm{a}} / 47.6^{\mathrm{b}} \\
(48.9 \pm 1.8)\end{array}$ & $\begin{array}{r}72.0^{\mathrm{a}} / 68.0^{\mathrm{b}} \\
(70.0 \pm 2.8)\end{array}$ & $\begin{array}{l}8.0^{\mathrm{a}} / 5.0^{\mathrm{b}} \\
(6.5 \pm 2.1)\end{array}$ \\
\hline MAR. & $\begin{array}{r}14.7^{\mathrm{a}} / 14.5^{\mathrm{b}} \\
(14.6 \pm 0.1)\end{array}$ & $\begin{array}{l}0.66^{\mathrm{a}} / 0.59^{\mathrm{b}} \\
(0.63 \pm 0.05)\end{array}$ & $\begin{array}{l}7.9^{\mathrm{a}} / 7.8^{\mathrm{b}} \\
(7.9 \pm 0.1)\end{array}$ & $\begin{array}{r}11.0^{\mathrm{a}} / 10.5^{\mathrm{b}} \\
(10.8 \pm 0.4)\end{array}$ & $\begin{array}{r}47.4^{\mathrm{a}} / 44.8^{\mathrm{b}} \\
(46.1 \pm 1.8)\end{array}$ & $\begin{array}{r}56.0^{\mathrm{a}} / 62.0^{\mathrm{b}} \\
(59.0 \pm 4.2)\end{array}$ & $\begin{array}{r}14.0^{\mathrm{a}} / 12.0^{\mathrm{b}} \\
(13.0 \pm 1.4)\end{array}$ \\
\hline APR. & $\begin{array}{c}16.2^{\mathrm{a}} / 16.3^{\mathrm{b}} \\
(16.3 \pm 0.1)\end{array}$ & $\begin{array}{l}0.73^{\mathrm{a}} / 0.78^{\mathrm{b}} \\
(0.76 \pm 0.04)\end{array}$ & $\begin{array}{l}7.6^{\mathrm{a}} / 7.9^{\mathrm{b}} \\
(7.8 \pm 0.2)\end{array}$ & $\begin{array}{l}10.8^{\mathrm{a}} / 9.6^{\mathrm{b}} \\
(10.2 \pm 0.8)\end{array}$ & $\begin{array}{c}36.0^{\mathrm{a}} / 42.2^{\mathrm{b}} \\
(39.1 \pm 4.4)\end{array}$ & $\begin{array}{r}54.0^{\mathrm{a}} / 60.0^{\mathrm{b}} \\
(57.0 \pm 4.2)\end{array}$ & $\begin{array}{r}14.0^{\mathrm{a}} / 16.0^{\mathrm{b}} \\
(15.0 \pm 1.4)\end{array}$ \\
\hline MAY & $\begin{array}{r}16.3^{\mathrm{a}} / 18.1^{\mathrm{b}} \\
(17.2 \pm 1.3)\end{array}$ & $\begin{array}{l}0.98^{\mathrm{a}} / 0.97^{\mathrm{b}} \\
(0.98 \pm 0.01)\end{array}$ & $\begin{array}{l}7.8^{\mathrm{a}} / 7.9^{\mathrm{b}} \\
(7.9 \pm 0.1)\end{array}$ & $\begin{array}{l}9.3^{\mathrm{a}} / 9.2^{\mathrm{b}} \\
(9.3 \pm 0.1)\end{array}$ & $\begin{array}{r}38.2^{\mathrm{a}} / 39.6^{\mathrm{b}} \\
(38.9 \pm 1.0)\end{array}$ & $\begin{array}{r}52.0^{\mathrm{a}} / 58.0^{\mathrm{b}} \\
(55.0 \pm 4.2)\end{array}$ & $\begin{array}{r}18.0^{\mathrm{a}} / 20.0^{\mathrm{b}} \\
(19.0 \pm 1.4)\end{array}$ \\
\hline JUN. & $\begin{array}{r}16.5^{\mathrm{a}} / 21.5^{\mathrm{b}} \\
(19.0 \pm 3.5)\end{array}$ & $\begin{array}{l}1.24^{\mathrm{a}} / 1.28^{\mathrm{b}} \\
(1.26 \pm 0.03)\end{array}$ & $\begin{array}{l}7.4^{\mathrm{a}} / 7.4^{\mathrm{b}} \\
(7.4 \pm 0.0)\end{array}$ & $\begin{array}{l}9.0^{\mathrm{a}} / 9.0^{\mathrm{b}} \\
(9.0 \pm 0.0)\end{array}$ & $\begin{array}{r}32.0^{\mathrm{a}} / 32.4^{\mathrm{b}} \\
(32.2 \pm 0.3)\end{array}$ & $\begin{array}{r}58.0^{\mathrm{a}} / 56.0^{\mathrm{b}} \\
(57.0 \pm 1.4)\end{array}$ & $\begin{array}{r}34.0^{\mathrm{a}} / 32.0^{\mathrm{b}} \\
(33.0 \pm 1.4) \\
\end{array}$ \\
\hline JUL. & $\begin{array}{r}16.4^{\mathrm{a}} / 20.1^{\mathrm{b}} \\
(18.3 \pm 2.6)\end{array}$ & $\begin{array}{l}1.79^{\mathrm{a}} / 1.61^{\mathrm{b}} \\
(1.70 \pm 0.13)\end{array}$ & $\begin{array}{l}7.3^{\mathrm{a}} / 7.2^{\mathrm{b}} \\
(7.3 \pm 0.1)\end{array}$ & $\begin{array}{l}9.1^{\mathrm{a}} / 9.0^{\mathrm{b}} \\
(9.1 \pm 0.1)\end{array}$ & $\begin{array}{r}32.0^{\mathrm{a}} / 34.2^{\mathrm{b}} \\
(33.1 \pm 1.6)\end{array}$ & $\begin{array}{r}62.0^{\mathrm{a}} / 60.0^{\mathrm{b}} \\
(61.0 \pm 1.4)\end{array}$ & $\begin{array}{r}38.0^{\mathrm{a}} / 36.0^{\mathrm{b}} \\
(37.0 \pm 1.4)\end{array}$ \\
\hline AUG. & $\begin{array}{r}15.8^{\mathrm{a}} / 16.9^{\mathrm{b}} \\
(16.4 \pm 0.8)\end{array}$ & $\begin{array}{l}1.77^{\mathrm{a}} / 1.58^{\mathrm{b}} \\
(1.68 \pm 0.13)\end{array}$ & $\begin{array}{l}7.6^{\mathrm{a}} / 7.5^{\mathrm{b}} \\
(7.6 \pm 0.1)\end{array}$ & $\begin{array}{l}9.0^{\mathrm{a}} / 9.1^{\mathrm{b}} \\
(9.1 \pm 0.1)\end{array}$ & $\begin{array}{r}34.2^{\mathrm{a}} / 32.8^{\mathrm{b}} \\
(33.5 \pm 1.0)\end{array}$ & $\begin{array}{r}64.0^{\mathrm{a}} / 62.0^{\mathrm{b}} \\
(63.0 \pm 1.4)\end{array}$ & $\begin{array}{l}78.0^{\mathrm{a}} / 60.0^{\mathrm{b}} \\
(69.0 \pm 12.7)\end{array}$ \\
\hline SEP. & $\begin{array}{r}14.2^{\mathrm{a}} / 16.4^{\mathrm{b}} \\
(15.3 \pm 1.6)\end{array}$ & $\begin{array}{l}1.65^{\mathrm{a}} / 1.56^{\mathrm{b}} \\
(1.61 \pm 0.06)\end{array}$ & $\begin{array}{l}7.9^{\mathrm{a}} / 7.8^{\mathrm{b}} \\
(7.9 \pm 0.1)\end{array}$ & $\begin{array}{l}9.2^{\mathrm{a}} / 9.1^{\mathrm{b}} \\
(9.2 \pm 0.1)\end{array}$ & $\begin{array}{c}32.2^{\mathrm{a}} / 35.0^{\mathrm{b}} \\
(33.6 \pm 2.0)\end{array}$ & $\begin{array}{r}60.0^{\mathrm{a}} / 64.0^{\mathrm{b}} \\
(62.0 \pm 2.8)\end{array}$ & $\begin{array}{l}78.0^{\mathrm{a}} / 55.0^{\mathrm{b}} \\
(66.5 \pm 16.3)\end{array}$ \\
\hline OCT. & $\begin{array}{r}14.0^{\mathrm{a}} / 13.6^{\mathrm{b}} \\
(13.8 \pm 0.3)\end{array}$ & $\begin{array}{l}0.98^{\mathrm{a}} / 0.96^{\mathrm{b}} \\
(0.97 \pm 0.01)\end{array}$ & $\begin{array}{l}7.8^{\mathrm{a}} / 7.6^{\mathrm{b}} \\
(7.7 \pm 0.1)\end{array}$ & $\begin{array}{r}12.9^{\mathrm{a}} / 11.5^{\mathrm{b}} \\
(12.2 \pm 1.0)\end{array}$ & $\begin{array}{r}40.0^{\mathrm{a}} / 44.4^{\mathrm{b}} \\
(42.2 \pm 3.1)\end{array}$ & $\begin{array}{r}68.0^{\mathrm{a}} / 66.0^{\mathrm{b}} \\
(67.0 \pm 1.4)\end{array}$ & $\begin{array}{r}38.0^{\mathrm{a}} / 34.0^{\mathrm{b}} \\
(36.0 \pm 2.8)\end{array}$ \\
\hline
\end{tabular}

(WT $=$ Water temperature, $\mathrm{DO}=$ Dissolved oxygen, $\mathrm{TA}=$ Total alkalinity, $\mathrm{TH}=$ Total hardness, $\mathrm{CV}=$ Current velocity, $\mathrm{T}=$ Turbidity ${ }^{\mathrm{a}}=$ Physico-chemical parameters during the year $2018-19,{ }^{\mathrm{b}}=$ Physico-chemical parameters during the year 2019-20) 
During this period it was found that the water temperature was maximum $(19.0 \pm 3.5)$ in the month of June and minimum $(10.8 \pm 0.5)$ in January. The velocity of water current was maximum (1.70 \pm 0.13$)$ during July and minimum $(0.59 \pm 0.06)$ in January. The $\mathrm{pH}$ and DO was maximum $(8.3 \pm 0.1)(13.2 \pm 0.2)$ in the month of January whereas $\mathrm{pH}$ was minimum $(7.3 \pm 0.1)$ in July and DO (9.0 \pm 0.0$)$ in June. The total alkalinity was maximum $(63.5 \pm 1.8)$ in the month January and minimum $(32.2 \pm 0.3)$ in June. The total hardness was recorded highest $(76.0 \pm 2.8)$ during

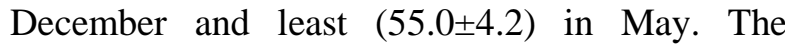
turbidity was least $(6.5 \pm 0.7)(6.5 \pm 2.1)$ in January and February whereas highest $(69.0 \pm 12.7)$ in August. Uehlinger et. al. (2010) found that the turbidity was high in glacial streams. The $\mathrm{pH}$ was found maximum during winter months due to algal growth. Similar results were also found by Dobriyal and Singh (1988) and Singh et. al. (1994) and Koshal et. al., (2016) in different rivers of Uttarakhand respectively.

Table 2 Nymph of macrozoobenthic communities (units $/ \mathrm{m}^{2}$ ) in the stream Balkhila at Tilfara during the year 2018-19 and 2019-20.

\begin{tabular}{|c|c|c|c|c|c|c|}
\hline Ephemeroptera & $\begin{array}{l}\text { November } \\
\text { December }\end{array}$ & $\begin{array}{l}\text { January } \\
\text { February }\end{array}$ & $\begin{array}{l}\text { March } \\
\text { April }\end{array}$ & $\begin{array}{l}\text { May } \\
\text { June }\end{array}$ & $\begin{array}{l}\text { July } \\
\text { August }\end{array}$ & $\begin{array}{l}\text { September } \\
\text { October }\end{array}$ \\
\hline Baetis & $\begin{array}{l}(32.3 \pm 15.3) \\
(53.9 \pm 15.2)\end{array}$ & $\begin{array}{l}59.2 \pm 7.6) \\
(43.1 \pm 15.2)\end{array}$ & $\begin{array}{l}(37.7 \pm 7.6) \\
(32.3 \pm 0.0)\end{array}$ & $\begin{array}{l}(16.2 \pm 7.6) \\
(0.0 \pm 0.0)\end{array}$ & $\begin{array}{l}(0.0 \pm 0.0) \\
(0.0 \pm 0.0)\end{array}$ & $\begin{array}{l}(5.4 \pm 7.6) \\
(16.2 \pm 7.6)\end{array}$ \\
\hline Cinygmula & $\begin{array}{l}(32.3 \pm 15.3) \\
(53.8 \pm 0.0)\end{array}$ & $\begin{array}{l}(59.2 \pm 22.8) \\
(59.2 \pm 53.3)\end{array}$ & $\begin{array}{l}(16.2 \pm 7.6) \\
(43.1 \pm 30.5)\end{array}$ & $\begin{array}{l}(32.3 \pm 15.3) \\
(16.2 \pm 7.6)\end{array}$ & $\begin{array}{l}(0.0 \pm 0.0) \\
(5.4 \pm 7.6)\end{array}$ & $\begin{array}{l}(21.6 \pm 15.2) \\
(16.2 \pm 7.6)\end{array}$ \\
\hline Caenis & $\begin{array}{c}(16.2 \pm 7.6) \\
(32.3 \pm 0.0)\end{array}$ & $\begin{array}{l}(16.2 \pm 22.8) \\
(16.2 \pm 7.6)\end{array}$ & $\begin{array}{l}(16.2 \pm 22.8) \\
(10.8 \pm 15.3)\end{array}$ & $\begin{array}{l}(5.4 \pm 7.6) \\
(0.0 \pm 0.0)\end{array}$ & $\begin{array}{l}(0.0 \pm 0.0) \\
0.0 \pm 0.0)\end{array}$ & $\begin{array}{l}(0.0 \pm 0.0) \\
(10.8 \pm 0.0)\end{array}$ \\
\hline Heptagenia & $\begin{array}{l}(21.6 \pm 15.2) \\
(27.0 \pm 22.8) \\
\end{array}$ & $\begin{array}{l}(32.3 \pm 0.0) \\
(43.1 \pm 45.6) \\
\end{array}$ & $\begin{array}{l}(16.2 \pm 7.6) \\
(24.3 \pm 11.4) \\
\end{array}$ & $\begin{array}{l}(5.4 \pm 7.6) \\
(0.0 \pm 0.0) \\
\end{array}$ & $\begin{array}{l}(0.0 \pm 0.0) \\
(0.0 \pm 0.0) \\
\end{array}$ & $\begin{array}{l}(0.0 \pm 0.0) \\
(26.9 \pm 7.6)\end{array}$ \\
\hline Total & $\begin{array}{l}(10.2 .3 \pm 22.8) \\
(166.9 \pm 7.6)\end{array}$ & $\begin{array}{l}(166.9 \pm 7.6) \\
(161.5 \pm 106.6)\end{array}$ & $\begin{array}{l}(86.2 \pm 45.6) \\
(110.4 \pm 26.7)\end{array}$ & $\begin{array}{l}(59.3 \pm 7.6) \\
(16.2 \pm 7.6)\end{array}$ & $\begin{array}{l}(0.0 \pm 0.0) \\
(5.4 \pm 7.6)\end{array}$ & $\begin{array}{l}(26.9 \pm 7.6) \\
(70.0 \pm 22.8)\end{array}$ \\
\hline \multicolumn{7}{|l|}{ Odonata } \\
\hline Euphaea & $\begin{array}{l}(5.4 \pm 7.6) \\
(0.0 \pm 0.0) \\
\end{array}$ & $\begin{array}{l}(5.4 \pm 7.6) \\
(0.0 \pm 0.0) \\
\end{array}$ & $\begin{array}{l}(0.0 \pm 0.0) \\
(0.0 \pm 0.0) \\
\end{array}$ & $\begin{array}{l}(0.0 \pm 0.0) \\
(5.4 \pm 7.6) \\
\end{array}$ & $\begin{array}{l}(0.0 \pm 0.0) \\
(0.0 \pm 0.0) \\
\end{array}$ & $\begin{array}{l}(0.0 \pm 0.0) \\
(0.0 \pm 0.0) \\
\end{array}$ \\
\hline Total & $\begin{array}{l}(5.4 \pm 7.6) \\
(0.0 \pm 0.0)\end{array}$ & $\begin{array}{l}(5.4 \pm 7.6) \\
(0.0 \pm 0.0)\end{array}$ & $\begin{array}{l}(0.0 \pm 0.0) \\
(0.0 \pm 0.0)\end{array}$ & $\begin{array}{l}(0.0 \pm 0.0) \\
(5.4 \pm 7.6)\end{array}$ & $\begin{array}{l}(0.0 \pm 0.0) \\
(0.0 \pm 0.0)\end{array}$ & $\begin{array}{l}(0.0 \pm 0.0) \\
(0.0 \pm 0.0)\end{array}$ \\
\hline \multicolumn{7}{|l|}{ Plecoptera } \\
\hline Perla & $\begin{array}{l}(5.4 \pm 7.6) \\
(10.8 \pm 15.2)\end{array}$ & $\begin{array}{l}(16.2 \pm 22.8) \\
(10.8 \pm 15.2)\end{array}$ & $\begin{array}{l}(16.2 \pm 22.8) \\
(8.1 \pm 11.5)\end{array}$ & $\begin{array}{l}(0.0 \pm 0.0) \\
(0.0 \pm 0.0)\end{array}$ & $\begin{array}{l}(0.0 \pm 0.0) \\
(0.0 \pm 0.0)\end{array}$ & $\begin{array}{l}(16.2 \pm 7.6) \\
(16.2 \pm 7.6)\end{array}$ \\
\hline Total & $\begin{array}{l}(5.4 \pm 7.6) \\
(10.8 \pm 15.2)\end{array}$ & $\begin{array}{l}(16.2 \pm 22.8) \\
(10.8 \pm 15.2)\end{array}$ & $\begin{array}{l}(16.2 \pm 22.8) \\
(8.1 \pm 11.5)\end{array}$ & $\begin{array}{l}(0.0 \pm 0.0) \\
(0.0 \pm 0.0)\end{array}$ & $\begin{array}{l}(0.0 \pm 0.0) \\
(0.0 \pm 0.0)\end{array}$ & $\begin{array}{l}(16.2 \pm 7.6) \\
(16.2 \pm 7.6)\end{array}$ \\
\hline Total nymph & $\begin{array}{l}(113.1 \pm 38.0) \\
(177.7 \pm 22.8)\end{array}$ & $\begin{array}{l}(188.4 \pm 22.9) \\
(172.2 \pm 91.4)\end{array}$ & $\begin{array}{l}(102.3 \pm 68.4) \\
(118.5 \pm 15.2)\end{array}$ & $\begin{array}{l}(59.3 \pm 7.6) \\
(21.6 \pm 15.2)\end{array}$ & $\begin{array}{l}(0.0 \pm 0.0) \\
(5.4 \pm 7.6)\end{array}$ & $\begin{array}{l}(43.1 \pm 15.2) \\
(86.2 \pm 30.3)\end{array}$ \\
\hline
\end{tabular}

Nymph and larvae of macrozoobenthos (units $/ \mathrm{m}^{2}$ ) in the stream Balkhila at Tilfara during the year 2018-19 and 2019-20 is presented in the Table 2 and 3. The stream is represented by 15 genera belonging to 6 orders. The benthic biota includes nymph and larvae of macrozoobenthos. The nymph mostly includes Ephemeroptera (Baetis, Cinygmula, Caenis, and Heptagenia), Odonata (Euphaea) and Plecoptera (Perla). The larvae includes Trichoptera (Glossosoma, Rhyachophila, Agapetus, Hydropsyche, Chimarra and Stenopsyche), Diptera (Antocha and Chironomus) and Coleoptera (Psephenus). The maximum numbers of macrozoobenthos were reported in the month of February $\left(387.5 \pm 106.5\right.$ units $\left./ \mathrm{m}^{2}\right)$ and January $\quad\left(382.2 \pm 144.7 \quad\right.$ units $\left./ \mathrm{m}^{2}\right)$, whereas, minimum in the month of July $(21.6 \pm 15.3$ units $\left./ \mathrm{m}^{2}\right)$ and August $\left(21.6 \pm 0.0\right.$ units $\left./ \mathrm{m}^{2}\right)$. During winter months all the physico-chemical factors were optimum which favored the growth of macrozoobenthos while during monsoon harsh environment restricted their growth. Rautela et. al. (2006) also found the similar trends of variation in macrozoobenthic density in the Khoh river of Garhwal Himalaya. The current velocity of water, temperature and dissolved substances are the 
important factors which regulates the distribution of benthic invertebrates (Siraj, 2018).

Regression analysis between physico-chemical parameters and the total macrozoobenthos of the stream Balkhila at Tilfara during the year 2018-19 and 2019-20 is presented in the Figure 2 to 5. The water temperature $\left(R^{2}=0.7378, r=-0.86\right)$ and the current velocity of water $\left(R^{2}=0.8371, r=-0.91\right)$ showed negative relationship with total macrozoobenthos. The dissolved oxygen $\left(\mathrm{R}^{2}=\right.$
0.7077, $\mathrm{r}=0.84)$ and $\mathrm{pH}\left(\mathrm{R}^{2}=0.8154, \mathrm{r}=0.90\right)$ showed positive relationship with total macrozoobenthos.

Tariq et. al. (2020) also found that water temperature and current velocity showed negative relationship whereas, dissolved oxygen and $\mathrm{pH}$ showed positive relationship with macrozoobenthic population.

Table 3: Larvae of macrozoobenthic communities (units $/ \mathrm{m}^{2}$ ) in the stream Balkhila at Tilfara during the year 2018-19 and 2019-20

\begin{tabular}{|c|c|c|c|c|c|c|}
\hline Trichoptera & $\begin{array}{l}\text { November } \\
\text { December }\end{array}$ & $\begin{array}{l}\text { January } \\
\text { February }\end{array}$ & $\begin{array}{l}\text { March } \\
\text { April }\end{array}$ & $\begin{array}{l}\text { May } \\
\text { June }\end{array}$ & $\begin{array}{l}\text { July } \\
\text { August }\end{array}$ & $\begin{array}{l}\text { September } \\
\text { October }\end{array}$ \\
\hline Glossosoma & $\begin{array}{l}(16.2 \pm 7.6) \\
(26.9 \pm 7.6) \\
\end{array}$ & $\begin{array}{l}(10.8 \pm 15.2) \\
(10.8 \pm 15.2) \\
\end{array}$ & $\begin{array}{l}(16.2 \pm 7.6) \\
(8.1 \pm 11.5) \\
\end{array}$ & $\begin{array}{l}(5.4 \pm 7.6) \\
(0.0 \pm 0.0) \\
\end{array}$ & $\begin{array}{l}(0.0 \pm 0.0) \\
(0.0 \pm 0.0) \\
\end{array}$ & $\begin{array}{l}(0.0 \pm 0.0) \\
(16.2 \pm 7.6) \\
\end{array}$ \\
\hline Rhyachophila & $\begin{array}{l}(21.6 \pm 15.2) \\
(21.6 \pm 30.5)\end{array}$ & $\begin{array}{l}(21.6 \pm 30.5) \\
(16.2 \pm 22.8)\end{array}$ & $\begin{array}{l}(37.7 \pm 38.0) \\
(5.4 \pm 7.6)\end{array}$ & $\begin{array}{l}(5.4 \pm 7.6) \\
(5.4 \pm 7.6) \\
\end{array}$ & $\begin{array}{l}(0.0 \pm 0.0) \\
(5.4 \pm 7.6) \\
\end{array}$ & $\begin{array}{l}(0.0 \pm 0.0) \\
(5.4 \pm 7.6) \\
\end{array}$ \\
\hline Agapetus & $\begin{array}{l}(5.4 \pm 7.6) \\
(0.0 \pm 0.0)\end{array}$ & $\begin{array}{l}(0.0 \pm 0.0) \\
(5.4 \pm 7.6)\end{array}$ & $\begin{array}{l}(0.0 \pm 0.0) \\
(0.0 \pm 0.0)\end{array}$ & $\begin{array}{l}(0.0 \pm 0.0) \\
(0.0 \pm 0.0)\end{array}$ & $\begin{array}{l}(0.0 \pm 0.0) \\
(0.0 \pm 0.0)\end{array}$ & $\begin{array}{l}(0.0 \pm 0.0) \\
(10.8 \pm 0.0)\end{array}$ \\
\hline Hydropsyche & $\begin{array}{l}(26.9 \pm 7.6) \\
(43.1 \pm 15.2) \\
\end{array}$ & $\begin{array}{l}(32.3 \pm 15.3) \\
(43.1 \pm 30.5) \\
\end{array}$ & $\begin{array}{l}(48.5 \pm 22.8) \\
(16.2 \pm 7.6) \\
\end{array}$ & $\begin{array}{l}(16.2 \pm 7.6) \\
(10.8 \pm 0.0) \\
\end{array}$ & $\begin{array}{l}(5.4 \pm 7.6) \\
(5.4 \pm 7.6) \\
\end{array}$ & $\begin{array}{l}(16.2 \pm 7.6) \\
(16.2 \pm 7.6) \\
\end{array}$ \\
\hline Chimarra & $\begin{array}{l}(16.2 \pm 22.8) \\
(10.8 \pm 15.2)\end{array}$ & $\begin{array}{l}(21.6 \pm 30.5) \\
(43.1 \pm 15.2)\end{array}$ & $\begin{array}{l}(21.5 \pm 0.0) \\
(10.8 \pm 15.2)\end{array}$ & $\begin{array}{l}(16.2 \pm 7.6) \\
(0.0 \pm 0.0)\end{array}$ & $\begin{array}{l}(0.0 \pm 0.0) \\
(0.0 \pm 0.0)\end{array}$ & $\begin{array}{l}(5.4 \pm 7.6) \\
(10.8 \pm 15.2)\end{array}$ \\
\hline Stenopsyche & $\begin{array}{l}(32.3 \pm 15.3) \\
(37.7 \pm 22.8)\end{array}$ & $\begin{array}{l}(16.2 \pm 22.8) \\
(48.5 \pm 7.6)\end{array}$ & $\begin{array}{l}(16.2 \pm 22.8) \\
(45.8 \pm 26.7)\end{array}$ & $\begin{array}{l}(37.7 \pm 22.8) \\
(10.8 \pm 15.2)\end{array}$ & $\begin{array}{l}(5.4 \pm 7.6) \\
(5.4 \pm 7.6) \\
\end{array}$ & $\begin{array}{l}(16.2 \pm 7.6) \\
(21.6 \pm 15.2)\end{array}$ \\
\hline Total & $\begin{array}{l}(118.5 \pm 30.3) \\
(139.9 \pm 15.3) \\
\end{array}$ & $\begin{array}{l}(102.3 \pm 83.7) \\
(166.9 \pm 7.6) \\
\end{array}$ & $\begin{array}{l}(140.0 \pm 30.5) \\
(86.2 \pm 15.2) \\
\end{array}$ & $\begin{array}{l}(80.8 \pm 7.6) \\
(27.0 \pm 22.8) \\
\end{array}$ & $\begin{array}{l}(10.8 \pm 0.0) \\
(16.2 \pm 7.6) \\
\end{array}$ & $\begin{array}{l}(37.7 \pm 7.6) \\
(80.8 \pm 7.6) \\
\end{array}$ \\
\hline \multicolumn{7}{|l|}{ Dipter } \\
\hline Antocha & $\begin{array}{l}(5.4 \pm 7.6) \\
(0.0 \pm 0.0) \\
\end{array}$ & $\begin{array}{l}(16.2 \pm 7.6) \\
(0.0 \pm 0.0) \\
\end{array}$ & $\begin{array}{l}(5.4 \pm 7.6) \\
(2.7 \pm 3.8) \\
\end{array}$ & $\begin{array}{l}(0.0 \pm 0.0) \\
(0.0 \pm 0.0) \\
\end{array}$ & $\begin{array}{l}(0.0 \pm 0.0) \\
(0.0 \pm 0.0) \\
\end{array}$ & $\begin{array}{l}(0.0 \pm 0.0) \\
(5.4 \pm 7.6) \\
\end{array}$ \\
\hline Chironomus & $\begin{array}{l}(16.2 \pm 7.6) \\
(21.6 \pm 15.2)\end{array}$ & $\begin{array}{l}(21.6 \pm 30.5) \\
(21.6 \pm 30.5)\end{array}$ & $\begin{array}{l}(16.2 \pm 7.6) \\
(2.7 \pm 3.8)\end{array}$ & $\begin{array}{l}(0.0 \pm 0.0) \\
(5.4 \pm 7.6)\end{array}$ & $\begin{array}{l}(5.4 \pm 7.6) \\
(0.0 \pm 0.0)\end{array}$ & $\begin{array}{l}(16.2 \pm 7.6) \\
(16.2 \pm 7.6)\end{array}$ \\
\hline Total & $\begin{array}{l}(21.6 \pm 15.2) \\
(21.6 \pm 15.2)\end{array}$ & $\begin{array}{l}(37.7 \pm 38.0) \\
(21.6 \pm 30.5)\end{array}$ & $\begin{array}{l}(21.6 \pm 0.1) \\
(5.4 \pm 7.6)\end{array}$ & $\begin{array}{l}(0.0 \pm 0.0) \\
(5.4 \pm 7.6)\end{array}$ & $\begin{array}{l}(5.4 \pm 7.6) \\
(0.0 \pm 0.0)\end{array}$ & $\begin{array}{l}(16.2 \pm 7.6) \\
(21.6 \pm 0.1)\end{array}$ \\
\hline \multicolumn{7}{|l|}{ Coleoptera } \\
\hline Psephenus & $\begin{array}{l}(21.6 \pm 30.5) \\
(37.7 \pm 7.6)\end{array}$ & $\begin{array}{l}(53.8 \pm 0.0) \\
(26.9 \pm 38.0)\end{array}$ & $\begin{array}{l}(16.2 \pm 22.8) \\
(18.9 \pm 19.0)\end{array}$ & $\begin{array}{l}(5.4 \pm 7.6) \\
(21.6 \pm 15.2)\end{array}$ & $\begin{array}{l}(5.4 \pm 7.6) \\
(0.0 \pm 0.0)\end{array}$ & $\begin{array}{l}(29.9 \pm 7.6) \\
(37.7 \pm 7.6) \\
\end{array}$ \\
\hline Total & $\begin{array}{l}(21.6 \pm 30.5) \\
(37.7 \pm 7.6) \\
\end{array}$ & $\begin{array}{l}(53.8 \pm 0.0) \\
(26.9 \pm 38.0) \\
\end{array}$ & $\begin{array}{l}(16.2 \pm 22.8) \\
(18.9 \pm 19.0) \\
\end{array}$ & $\begin{array}{l}(5.4 \pm 7.6) \\
(21.6 \pm 15.2) \\
\end{array}$ & $\begin{array}{l}(5.4 \pm 7.6) \\
(0.0 \pm 0.0) \\
\end{array}$ & $\begin{array}{l}(29.9 \pm 7.6) \\
(37.7 \pm 7.6) \\
\end{array}$ \\
\hline Total larvae & $\begin{array}{l}(161.6 \pm 15.1) \\
(199.2 \pm 22.8)\end{array}$ & $\begin{array}{l}(193.8 \pm 121.8) \\
(215.3 \pm 15.1)\end{array}$ & $\begin{array}{l}(177.7 \pm 53.2) \\
(110.5 \pm 3.9)\end{array}$ & $\begin{array}{l}(86.2 \pm 0.1) \\
(53.9 \pm 45.7)\end{array}$ & $\begin{array}{l}(21.6 \pm 15.3) \\
(16.2 \pm 7.6)\end{array}$ & $\begin{array}{l}(80.8 \pm 22.8) \\
(140.1 \pm 0.1)\end{array}$ \\
\hline Grand Total & $\begin{array}{l}(274.7 \pm 53.1) \\
(376.8 \pm 45.7) \\
\end{array}$ & $\begin{array}{l}(382.2 \pm 144.7) \\
(387.5 \pm 106.5) \\
\end{array}$ & $\begin{array}{l}(280.0 \pm 15.2) \\
(228.9 \pm 11.4) \\
\end{array}$ & $\begin{array}{l}(145.4 \pm 7.6) \\
(75.5 \pm 60.9) \\
\end{array}$ & $\begin{array}{l}(21.6 \pm 15.3) \\
(21.6 \pm 0.0) \\
\end{array}$ & $\begin{array}{l}(123.8 \pm 7.6) \\
(226.2 \pm 30.3)\end{array}$ \\
\hline
\end{tabular}




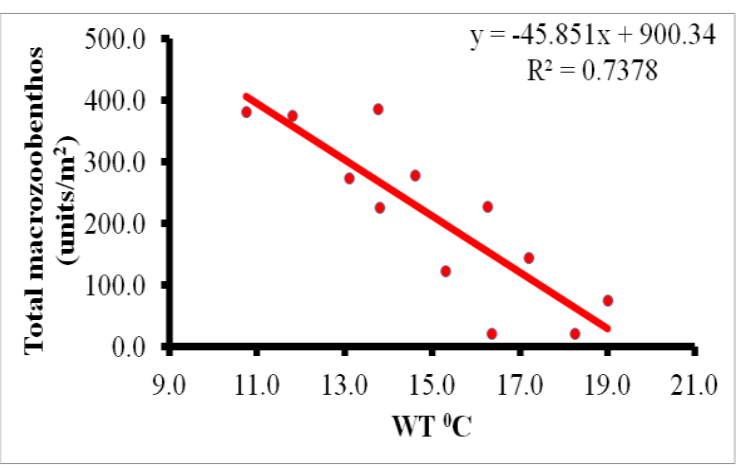

Figure 2: Regression analysis between water temperature and total macrozoobenthos of the stream Balkhila at Tilfara during the year 2018-19 and 2019-20.

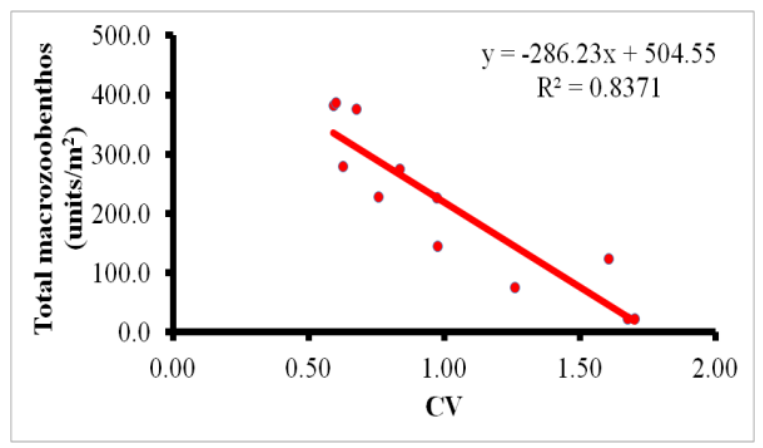

Figure 3: Regression analysis between current velocity and total macrozoobenthos of the stream Balkhila at Tilfara during the year 2018-19 and 2019-20.

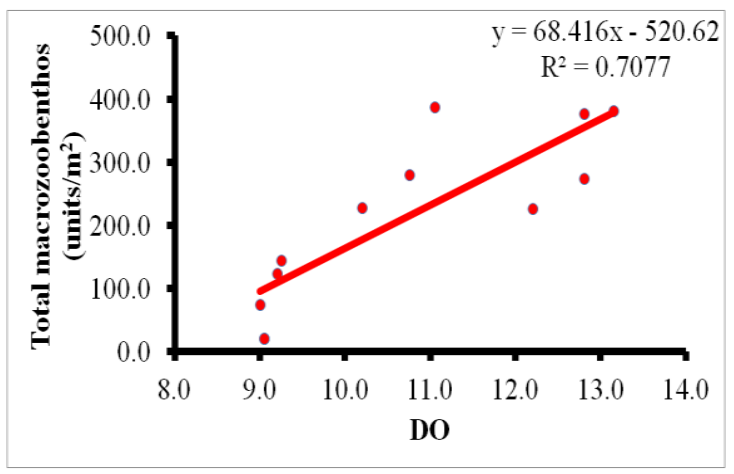

Figure 4: Regression analysis between dissolved oxygen and total macrozoobenthos of the stream Balkhila at Tilfara during the year 2018-19 and 2019-20.

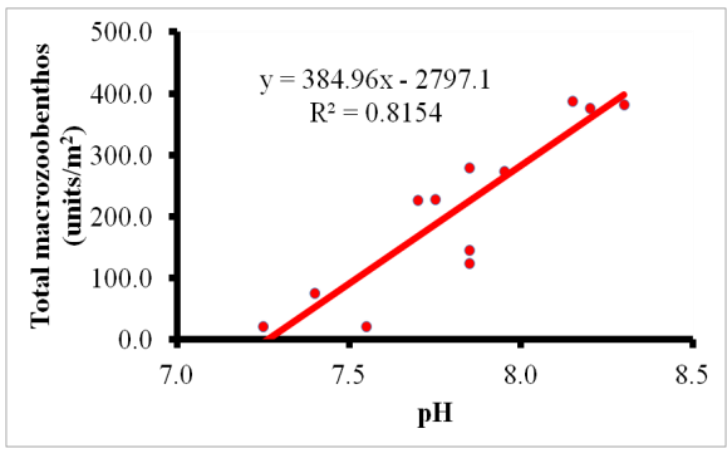

Figure 5: Regression analysis between $\mathrm{pH}$ and total macrozoobenthos of the stream Balkhila at Tilfara during the year 2018-19 and 2019-20.

Shannon-Wiener diversity index of macrozoobrnthos in the stream Balkhila at Tilfara during the year 2018-19 and 2019-20 is presented in Figure 6. The $\mathrm{H}^{\prime}$ was recorded maximum (2.56) in the month of November while minimum (1.39) during July and August. The index value is mostly high during winter months which suggested the stable ecosystem as all the parameters are in optimum condition whereas, least value in monsoon depicted the perturbed state of ecological setup. Habib and Yousuf (2012) also used Shannon-Wiener diversity index to study the diversity of macroinvertebrate community of Doodganga stream and Khanshah Manshah canal in Kashmir Himalaya, they found that high diversity value in Doodhganga upstream is the result of positive correlation between physicochemical parameters and the corresponding diversity while low diversity in KhanshahManshah downstream.

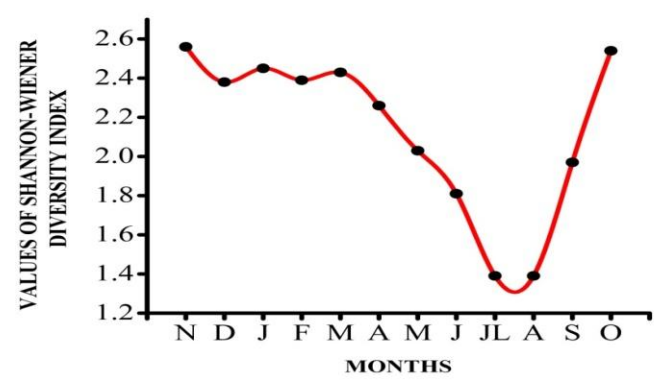

Figure 6: Shannon-Wiener diversity index of macrozoobrnthos in the stream Balkhila at Tilfara during the year 2018-19 and 2019-20. 
Multivariate cluster analysis of macrozoobenthic orders of the stream Balkhila at Tilfara during the year 2018-19 and 2019-20 is presented in Figure 7. The dendrogram formed thus suggested that the Ephemeroptera and Trichoptera were homogenous group which was later joined by least diverse homogenous group of Diptera, Coleoptera, Odonata and Plecoptera. Ramirez et al. (2009) also analysed the Cluster analysis of macroinvertebrate assemblage composition in 9 tributaries of the Turabo watershed. Adriaenssens et. al. (2007) also used the clustering techniques for ecological classification of series of sites in the Zwalm river basin (Flanders, Belgium).

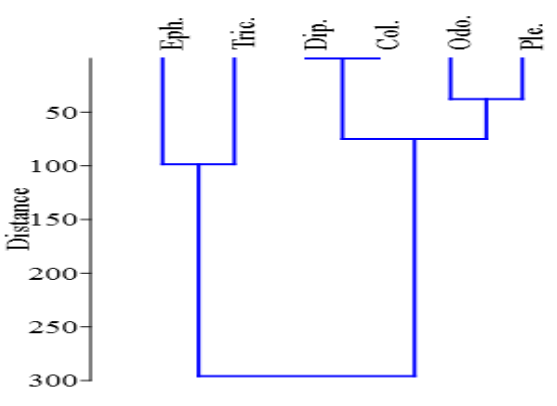

Figure 7: Multivariate cluster analysis of macrozoobenthic orders of the stream Balkhila at Tilfara during the year 2018-19 and 2019-20.
Acronyms $=$ Ephe- Ephemeroptera, TricTrichoptera, Dip- Dipterta, Col- Coleoptera, Odo- Odonata, Ple- plecoptera.

Canonical correspondence analysis (CCA) of macrozoobenthic orders of different seasons in the stream Balkhila at Tilfara during the year 2018-19 and 2019-20 is presented in Figure 8. CCA was performed to investigate the effect of different detrimental ecological factors on the characteristic orders of different seasons. CCA plot suggested that the winter and spring season was represented by most of the benthic orders Ephemeroptera Trichoptera, Plecoptera and Diptera and the factors governing them were dissolved oxygen, total alkalinity, $\mathrm{pH}$ and total hardness. Trichoptera was also associated with Autumn season whereas the summer season is represented by Odonata and the factor associated was water temperature. The monsoon season was sparsely represented by order Coleoptera and the major factors associated were current velocity and turbidity. Sagir, et. al. (2020) also used the CCA to study the benthic population in the Western Nayar river of Garhwal Himalaya. Kumar and Nautiyal (2019) used the CCA to study benthic macroinvertebrates at different sites in the Bhagirathi river.

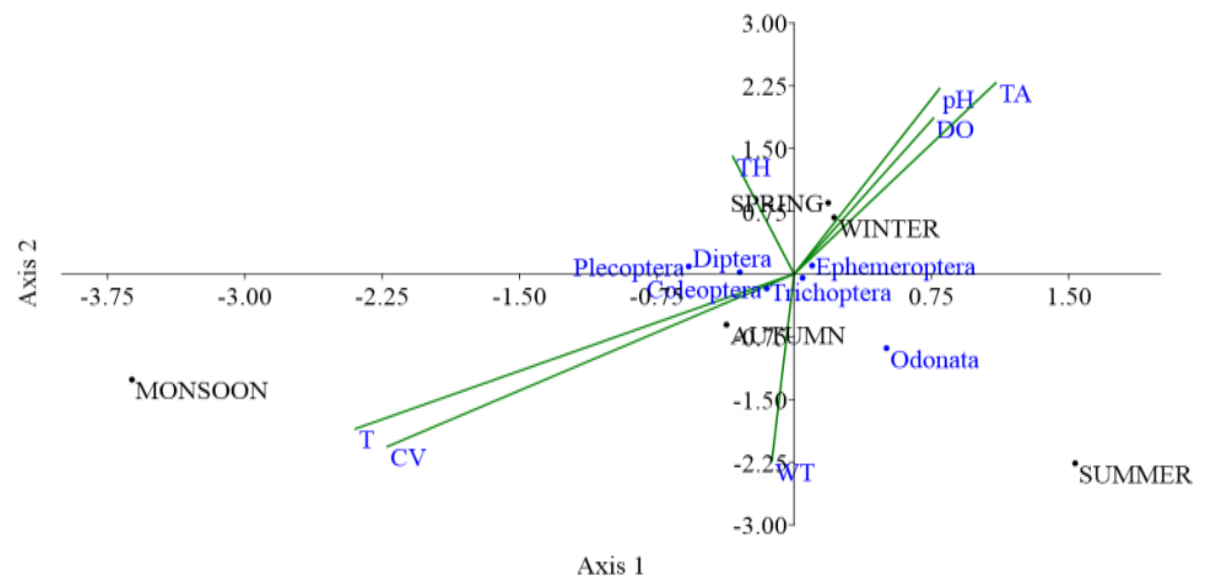

Figure 8: Canonical correspondence analysis (CCA) of macrozoobenthic orders of different seasons in the stream Balkhila at Tilfara during the year 2018-19 and 2019-20 
From the above discussion it is concluded that the winter season with supportive ecological conditions offers better ground for the benthic population to flourish well whereas, monsoon season with disturbed ecological setup showed least diversity.

\section{Acknowledgement}

Authors thankfully acknowledge the University Grant Commission for providing Junior Research Fellowship.

\section{References}

Adriaenssens V, Verdonschot PFM, Goethals PL $M$ and Pauw ND (2007) Application of clustering techniques for the characterization of macroinvertebrate communities to support river restoration management. Aquatic Ecology. 41:387-398.

APHA (2012) Standard methods for examination of water and wastewater. $22^{\text {nd }}$ Edn., APHA, AWWA, WPCF, Washington DC, USA.

Bahuguna BK, Nautiyal R, Nautiyal $P$ and Singh H R (2004) Stream regulation: Variation in the density, composition and diversity of benthic macroinvertebrates occurring in the up and downstream sections of the impounded zones of the river Ganga in the foothills. Trop. Ecol. 5: 251-261.

Bahuguna P, Negi S and Dobriyal AK (2019) Density and diversity of aquatic mites in a spring-fed stream of Garhwal Himalaya. $J$ Mountain Res. 14 (2): 57-61.

Balodi VP and Koshal Kumar(2015) MacroZoobenthic study in relation to physico Chemical parameters of Khoh River in Uttarakhand. Int. J. of Sci. Res. Vol.4 (6) 171-173.

Brittain, JE, Saltveit, SJ, Castella, E, Bogen, J, Bønsnes, TE, Blakar I, Bremnes T, Haug I and Velle G (2001) The macroinvertebrate communities of two contrasting Norwegian glacial rivers in relation to environmental variables. Freshwater Biology. 46: $1723-$ 1736.
Dobriyal AK, Balodi VP, Joshi HK, Thapliyal A, Bahuguna P, Uniyal SP and Kotnala CB (2009) Substratum heterogeneity and indicator macro-zoobenthos of the Eastern Nayar, Garhwal, Central Himalaya. $J$. Mountain Res. 4: 130-135.

Dobriyal AK, Singh H R (1988) Ecological basis for the ichthyofaunal variation in two hillstrteams of Garhwal Himalaya. In: M. Mohan Joseph (Ed.) The First Indian Fisheries Forum Proceedings, Asian Fishery Society, Indian Branch.313-317.

Edmondson T H (1992) (Ed). Freshwater Biology, Ward. H B and Whipple, G H, John Wiley and Sons, NY.

Habib S and Yousuf AR (2012) Benthic macroinvertebrate community of Yousmarg streams (Doodhganga stream and Khanshah Manshah canal) in Kashmir Himalaya. J. Ecol. Nat. 4(11): 280-289.

Heino J and Mykra H (2008) Control of stream insect assemblages: roles of spatial configuration and local environmental factors. Ecol. Entomol. 33: 614-622.

Hynes HBN (1970) The ecology of the running waters. Liverpool University Press. 543.

Ilg, C and Castella E (2006) Patterns of macroinvertebrate traits along three glacial stream continuums. Freshw. Biol. 51: 840853.

Jessup B K, Markowitz A, Stribling J B (2002). Family level key to the stream invertebrates of Maryland and surrounding areas. Tetra Tech Inc. 10045 Red Run Blvd. Suite.110, Owings Mills, MD. 21117-6102:80.

Katoch PD, Balodi VP , Thapliyal A, Kumar K, Rashid M, Sagir M and Dobriyal AK (2015) Population structure and diversity analysis of benthic Ephemeropterans in Western Nayar river during 2014-15, J. Mountain Res. Vol. 10, pp 55-66.

Koshal Kumar, Anita Rawat Rana and Kotnala CB (2016) Water Quality and Pollution Status of Rawasan Stream In Garhwal Himalaya, Uttarakhand, India. J Mountain Res, 11:9-14 
Koshal Kumar, Rana JS, Rana AR and Kotnala, CB (2017) Checklist of Benthic Macroinvertebrate Communities of Stream Rawasan In Garhwal Region (Central Himalaya), Uttarakhand (India). J. Mountain. Res 12: 91-95

Kumar S and Nautiyal P (2019) Community Features of Benthic Macroinvertebrates and Bioassessment of the Stretch of Bhagirathi River Impacted by Maneri Bhali Stage I Hydroelectric Project. International Journal of Ecology and Environmental Sciences. 45 (3): 311-315, 2019.

Milner AM, Taylor RC and Winterbourn MJ (2001) Longitudinal distribution of macroinvertebrates in two glacier-fed New Zealand rivers. Freshw. Biol. 46: 17651775.

Milner AM, Britain J, Castella E, Petts G, (2001) Trends of macroinvertebrate community structure in glacier-fed rivers in relation to environmental conditions: a synthesis. Freshw. Biol. 46 (12):1833-1847.

Rautela KK, Kumar K, Bisht K L, Joshi VD,Negi, K S, Rautela A S and Dobriyal A K (2006) Ecological studies on the biodiversity of river Khoh in the foot-hills of Garhwal Himalaya: Part II. Macrozoobenthic analysis. Aquacult, 7: 277-283

Ramirez A, Crespo RDJ, Cardona DMM, Rivera, NM and Caraballo SB (2009) Urban streams in Puerto Rico: what can we learn from the tropics? J. N. Am. Benthol. Soc. 28(4):10701079.

Rosenberg DM and Resh VH (1993) Freshwater biomonitoring and benthic macroinvertebrates, Chapman \& Hall, New York.

Saether O (1968) Chironomids from the Finse area, Norway, with special reference to their distribution in a glacier brook. Archiv fu" $r$ Hydrobiol. 64: 426-483.

Sagir M and Dobriyal AK (2020) Structural and function diversity periphyton community from the Western Nayar river of Garhwal Himalaya. Uttar Pradesh J Zool. 41(1): 1529.
Singh HR, Nautiyal P, Dobriyal AK, Pokhriyal RC, Negi M, Baduni V, Nautiyal R, Agarwal NK and Gautam A (1994). Water quality of the river Ganga (Garhwal Himalayas). Acta Hydrobiol. 36: 3-15.

Siraj S, (2018) Variations in species composition and distribution of macrozoobenthos altitudinal gradient in Sindh River of Kashmir Himalaya. JK Knows.Init. 2(1):97107.

Stanford JA and Ward J B (1983) Insect species diversity as a function of environmental variability and disturbance in streams: pp. 165-178. In: J.R. Barnes \& G.W. Minshall(eds.) Stream Ecology: Application and Testing of General Ecological Theory. Plenum Press, New York.

Steffan AW (1971) Chironomid (Diptera) biocoenoses in Scandinavian glacier brooks. Can. Entomol., 103: 477-486.

Tariq M Kotnala CB and Dobriyal AK (2020) Dynamics and diversity of macrozoobenthos in the glacier fed stream Balkhila at Siron in Chamoli Garhwal. Uttar Pradesh J Zool. 41(8):80-91.

Thompson R \& Townsend C (2006). A truce with neutral theory: local deterministic factors, species traits and dispersal limitation together determine patterns of diversity in stream invertebrates. J. Anim. Ecol.. 75: 476-484.

Uehlinger U, Robinson CT, Hieber M and Zah R (2010) The physico-chemical habitat template for periphyton in alpine glacial streams under a changing climate. Hydrobiologia. 657:107-121.

Welch PS (1948) Limnological methods. McGraw Hill, New York. 